\title{
Bone Cancer and Metastasis Trials, Drug Treatment
}

\section{Da-Yong Lu* and Bin Xu}

School of Life Sciences, Shanghai University, Shanghai, China

*Corresponding Author: Da-Yong Lu, School of Life Sciences, Shanghai University, Shanghai, China.
Received: July 31, 2021

Published: August 12, 2021

(C) All rights are reserved by Da-Yong Lu and

Bin Xu.

\begin{abstract}
Bone cancer and metastasis is difficult to be managed. In the early stage, amputation is widely practiced in the clinic. To avoid this devastating event, drug treatment should be strengthened. Several factors or associations should be noticed for therapeutic promotion and paradigms. This editorial discusses drug development and clinical treatment for bone cancer and metastasis.

Keywords: Bone Cancer; Neoplasm Metastasis; Drug Treatment; Drug Targets; Personalized Medicine
\end{abstract}

\section{Introduction}

Cancer is the secondary leading cause of human mortality worldwide [1-4]. Bone cancer and metastasis is one of frequent cancer categories and difficult to be managed by current medicine. In the early stage, amputation is widely practiced in the clinic. To avoid this devastating event, drug treatment should be a future trend.

Several factors or associations should be noticed for therapeutic promotion and paradigms. They contain the disciplines of diagnosis, pathophysiology, pharmacology and pharmaceuticals. This editorial discusses the landscape of drug development and clinical treatment for bone cancer and metastasis.

\section{Emerging problems}

Currently, anticancer drugs are proposed to be the foremost therapeutic selection by avoiding big inconvenience of radical therapy. There are a lot of different anticancer drugs in the clinic. How to optimize drug therapy is a key for promoting patient lifequality and survival. Facing with therapeutic setback of surgery and drug diversity, individualized drug therapy will play major role for therapeutic selection. Several pathways can improve these therapeutic responses and benefiting for bone cancer and metastasis treatment [5].
Major pathways

Basic study

- Identify and discover key genetic or molecular factors that is involving bone cancer and metastasis [6].

- Development of high-active anticancer or antimetastatic drugs [7-9].

- $\quad$ Drug targets and mechanisms should be searched [10,11].

Clinical study

- Herbal medicine may promote human physiological conditions and integrity [12-14].

- $\quad$ Personalized medicine is a drug selection paradigm that may optimize drug treatment against cancer growth and metastasis [15-25].

- Drug combination commonly promote clinical outcomes yet mechanisms should be understood $[26,27]$.

Future study

- Mathematics and computational aids [28-31].

- Pharmaceutical innovation, such as nanomedicine and other drug delivery systems [32]. 


\section{Discussion}

Novel approaches aiming at promoting drug therapy against growth and metastasis of bone cancers should be strengthened in the future. Moreover, drug targets, mechanisms, pharmacology, toxicity and medicinal chemistry should be separately or integrate compared.

\section{Conclusion}

Drug development for bone cancer and metastasis treatment should be indispensable. Many new pathways should be explored and promoted. Facing with important oncologic and pharmacological challenge, hard work will be paid of.

\section{Bibliography}

1. Lambert AW., et al. "Emerging biological principles of metastasis". Cell 168 (2017): 670-691.

2. Ahmad AS., et al. "Trends in the lifetime risk of developing cancer in Great Britain; Comparison of risk for those born from 1930-1960". British Journal of Cancer 112 (2015): 943-947.

3. Mehlen P and Puisieux A. "Metastasis; a question of life or death". Nature Reviews Cancer 6 (2006): 449-458.

4. Weidenfeld K and Barkan D. "EMT and stemness in tumor dormacy and outgrowth: Are they intertwined processes?" Frontiers in Oncology 8 (2018): 381.

5. Pantano F., et al. "Integrin alpha 5 in human breast cancer is a mediator of bone metastasis and a therapeutic target for the treatment of osteolytic lesions". Oncogene 40 (2021): 12841299.

6. Lu DY and Che JY. "Bone disease treatments, importance of technical supports". Acta Scientific Orthopaedics 4.4 (2021): 55-57.

7. Lu DY., et al. "Cancer Metastasis treatments". Current Drug Therapy 8.1 (2013): 24-29.

8. Lu DY., et al. "Anti-metastatic drug development, work out towards new direction". Medicinal Chemistry 8.7 (2018): 192196.

9. Lu DY and Lu TR. "Antimetastatic activities and mechanisms of bisdioxopiperazine compounds". Anti-Cancer Agent Medicinal Chemistry 10.7 (2010): 564-570.
10. Zhu H., et al. "DJ-1 mediates the resistance of cancer cells to dihydroarteminisinin through cancer cells through reactive oxygen species removal". Free Radical Biology and Medicine 71 (2014): 121-132.

11. Lu DY., et al. "Development of antimetastatic drugs by targeting tumor sialic acids". Scientia Pharmaceutica 80.3 (2012): 497-508.

12. Agarwal N., et al. "Natural herbs as anticancer drugs". Journal Pharm Tech, International Journal 4.3 (2012): 1142-1153.

13. Lu DY., et al. "Natural drug cancer treatment strategies from herbal medicine to chemical or biological drug". Studies in Natural Products Chemistry 66 (2020): 91-115.

14. Lu DY and Lu TR. "Herbal medicine in new era". Hospice Palliative Medicine International Journal 3.4 (2019): 125-130.

15. Lu DY., et al. "Individualized cancer chemotherapy integrating drug sensitivity tests, pathological profile analysis and computational coordination-an effective strategy to improve clinical treatment". Medical Hypotheses 66.1 (2006): 45-51.

16. Lu DY. "Personalized cancer chemotherapy, an effective way for enhancing outcomes in clinics. Woodhead Publishing, Elsevier UK (2014).

17. Volm M and Efferth T. "Prediction of cancer drug resistance and implications for personalized medicine". Frontiers in Oncology (2015): 282.

18. Lu DY., et al. "Anticancer drug sensitivity testing, a historical review and future perspectives". Current Drug Therapy 10.1 (2015): 44-55.

19. Lu DY and Lu TR. "Drug sensitivity testing, a unique drug selection strategy". Advances in Biomarker Sciences and Technology 2 (2020): 59-66.

20. Lu DY., et al. "Cancer bioinformatics for update anticancer drug developments and personalized therapeutics". Reviews on Recent Clinical Trials 12.2 (2017): 101-110.

21. Lu DY., et al. "Pharmacogenetics of cancer therapy: breakthroughs from beyond?" Future Science OA 1.4 (2015): 80.

22. Montero J., et al. "Drug-induced death signaling strategy rapidly predicts cancer response to chemotherapy". Cell 160 (2015): 977-989. 
23. Popova AA and Levkin PA. "Precision medicine in oncology: In vitro drug sensitivity and resistance test (DSRT) for selection of personalized anticancer therapy". Adv Therapeutics (2020): 1900100.

24. Hyman DH., et al. "Implementing genome-driven oncology". Cell 168 (2017): 584-599.

25. Lu DY., et al. "Individualized cancer therapy, future approaches". Current Pharmacogenomics and Personalized Medicine 16.2 (2018): 156-163.

26. Lu DY., et al. "Anticancer drug combination, how far we can go through?" Anti-Cancer Agents in Medicinal Chemistry 17.1 (2017): 21-28.

27. Lu DY., et al. "Drug combination in clinical cancer treatment". Reviews on Recent Clinical Trials 12.3 (2017): 202-211.

28. Franssen L., et al. "A mathematical framework for modeling the metastatic spread of cancer". Bulletin of Mathematical Biology 81 (2019): 1965-2010.

29. Gerlee $P$ and Johanson M. "Infering rates of metastatic dissemination using stochastic network models". PLOS computational Biology 15.4 (2019): e1006868.

30. Freedman DH. "Hunting for new drugs with AI". Nature 576 (2019): s49-s53.

31. Lu DY., et al. "Anatomic approaches for cancer metastatic study". EC Clinical and Experimental Anatomy 3.9 (2020): 3234.

32. Barani M., et al. "Recent advances in nanotechnology-based diagnosis and treatments of human osteosarcoma". Biosensors 11 (2021): 55.

Volume 4 Issue 9 September 2021

(C) All rights are reserved by Da-Yong Lu and Bin Xu. 